\title{
Über urnenfelderzeitliche Schmuckschnecken aus Nußdorf ob der Traisen (Niederösterreich)
}

\section{Einleitung}

Das Gebiet um Nußdorf ob der Traisen mit der Kat. Gem. Franzhausen im unteren Traisental (Niederösterreich) ist archäologisch überaus ergiebig:

Über die notenkopfkeramische Siedlung von Franzhausen, die 1993 beim Straßenbau entdeckt wurde, berichten u. a. BlesL (1995) und NeUGEBAUER (1997). Auf dem Niederterrassenvorsprung der Traisen befanden sich außerdem zwei zeitgleiche große Frühbronzezeit-Nekropolen: Franzhausen I (Grabungen 1981-1983) und Franzhausen II (gegraben zwischen 1985 und 1991; ab 1993 regelmäßig fortgesetzt). Im Nordwesten an Franzhausen II anschließend befindet sich eine kleinere Gräbergruppe der älteren Urnenfelderkultur. Im Südosten direkt an der Terrassenkante zur Traisen konnte weiters in den Jahren 1981-1982 und 1987 ein großes jungurnenfelderzeitliches Gräberfeld geborgen werden. Dieses wurde während Hallstatt B3 aufgegeben; zu Beginn von Hallstatt $\mathrm{C}$ erfolgte die Neuanlage eines eisenzeitlichen Friedhofes, der bis in Latène B belegt war (NEugEBauER 1997; auch 1992).

Dr. Michaela LochNer (Prähistorische Kommission der Österreichischen Akademie der Wissenschaften, Wien) übergab mir im Herbst 1999 Fundobjekte aus 18 Brandgräbern des Gräberfeldes der jüngeren Urnenfelderzeit zur Begutachtung ${ }^{1}$. Sieben Fundnummern enthielten Molluskenbeigaben; die übrigen 11 umfaßten kleine Knochenringe (Durchmesser unter $1 \mathrm{~cm}$ ) in Einoder Mehrzahl, die als Schmuck- oder Trachtbestandteile zu interpretieren sind. Da nur wenige dieser Knochenscheibchen Verkohlungsspuren zeigen und die Molluskenschalen fast alle in sehr gutem Erhaltungszustand sind, dürften die Objekte erst nach der Leichenverbrennung beigelegt worden sein.

Die Tatsache, daß die beiden reichhaltigsten Kontexte (Fnr. 11, Verf. 761, und Fnr. 6, Verf. 568) aus Landschnecken bestanden, die während der zur Diskussion stehenden Besiedlungszeit im Gebiet gelebt haben, sowie der zeitliche Horizont (Urnenfelderzeit) ließen diese Ergebnisse publikationswürdig erscheinen. Molluskenschalen als Schmuckobjekte oder Bestandteile der Tracht wurden in erster Linie vom paläolithischen und neolithischen Menschen verwendet, wobei teils Fossilien der näheren oder weiteren Umgebung, sogar aus relativ weit entfernten Vorkommensgebieten, Verwendung fanden, teils zeitgenössische marine Arten, die vorwiegend aus dem Mittelmeerraum herbeitransportiert worden sind.

\section{ERgebnisse}

Bei den Molluskenbeigaben handelt es sich um drei Arten von Landschnecken, die während der Belegungszeit des Gräberfeldes im Gebiet ge-

${ }^{1}$ Die Aufarbeitung des 411 Gräber umfassenden Bestattungsplatzes erfolgte im Rahmen eines FWF-Projektes sowie seit 1999 an der Prähistorischen Kommission der ÖAW durch Michaela Lochner. Eine erste zusammenfassende Darstellung siehe unter LOCHNER, M. (1994). 
lebt haben, sowie um zwei Arten von Süßwassermuscheln, die entweder aus der Traisen oder aus der Donau stammen und ebenfalls zeitgleich sind. Die ersteren zeigen eindeutige Bearbeitungsspuren in Form von Perforationen, die zwecks Auffädelns bzw. Aufnähens auf Kleidungsstücken gesetzt worden sind, die letzteren sind (mit einer Ausnahme?) unbearbeitet beigegeben.

Die zahlenmäßig vorherrschende Art ist Zebrina detrita (O. F. MüLLER 1774) (Buliminidae), die Märzen- oder Zebraschnecke, gegenwärtig meridional verbreitet. Bei den anderen Landschnecken handelt es sich um die Gerippte Bänderschnecke, Cepaea vindobonensis (A. FÉRUSSAC 1821) (Helicidae), ost- und südosteuropäisch verbreitet, und um die Dreizahn-Vielfraßschnecke, Chondrula tridens (O. F. MüLLER 1774), mittel-, ost- und südosteuropäisch verbreitet. Alle sind Bewohner warmer, trockener Rasenbiotope, Böschungen, xerothermer Buschlandschaften, auch von Weinbergen. Für alle Arten sind gegenwärtig Biotopentzug durch landwirtschaftliche Maßnahmen und Biotopvernichtung (z. B. Einsatz von Herbiziden, Insektiziden und Überdüngung) bestandsbedrohlich; für Chondrula tridens gilt in Niederösterreich und Gesamtösterreich Gefährdungsstufe 1, für Zebrina detrita gilt für Niederösterreich und Gesamtösterreich Gefährdungsstufe 2 (stark gefährdet), für Cepaea vindobonensis Stufe 3 (gefährdet); vgl. Frank \& ReISchüтz 1994: 299, 313; heutige Vorkommen in Österreich siehe KLEmM (1974: 183-185, Karte 49, 191-193, Karte 52, 466-469, Karte 152).

Die Großmuscheln (Unionidae) sind Unio pictorum (Linnaeus 1758), die Gemeine Malermuschel (europäisch; verschiedene Fließ- und Stehgewässer), und die Gemeine Flußmuschel, Unio crassus PhILIPsson (europäisch; saubere Bäche und Flüsse). Während der Fortbestand der ersteren in Österreich noch gewährleistet ist, befindet sich die zweitere an vielen ihrer mitteleuropäischen Standorte stark im Rückgang. Aus Österreich sind heute nur noch wenige Lebendvorkommen bekannt; vgl. REISCHÜTZ \& SACKL 1991; FranK \& REISCHÜTZ 1994: 313.

\subsection{Artenliste}

Buliminidae

Chondrula tridens (O. F. MüLLER 1774); Verf. 262.

Zebrina detrita (O. F. MüLLER 1774); Verf. 568, Verf. 761 (Abb. 2, 3, 4).

Helicidae

Cepaea vindobonensis (A. FÉRussaC 1821); Verf. 761 (Abb. 5, 6).

Unionidae

Unio pictorum (Linnaeus 1758); Verf. 74 (Abb. 14), Verf. 262, Verf. 662.

Unio crassus Philipsson 1788; Verf. 262 (cf.), Verf. 263, Verf. 723 (Abb. 1).

\subsection{Die Fundnummern}

Im Text werden die folgenden Abkürzungen verwendet: $\mathrm{B}=$ Schalenbreite, $\mathrm{D}=$ Dicke,$\quad$ Ex. $=$ Exemplar(e) $\quad$ Fnr. $=$ Fundnummer,$\quad$ Fo. $=$ Fundort $(\mathrm{e})$, Fragm. $=$ Fragment $(\mathrm{e}), \mathrm{H}=$ Schalenhöhe, $\mathrm{L}=$ Schalenlänge, Verf. $=$ Verfärbung.

Die anthropologischen Bestimmung des Leichenbrands erfolgte durch Dr. Silvia Renhart. 
Verf. 74, Fnr. 5 (Akte 260/81: „Muschel aus Urne - Fnr. 2“)

Unio pictorum: 2 Fragmente einer linken Klappe, dünnschalig (von einem Exemplar?)

Das größere Stück besteht aus Wirbel und Oberrand, Vorder- und Hinterende sowie Unterrand fehlen. Der Bruchrand unterhalb des Wirbels läßt eine ausgebrochene Perforation erkennen, die offenbar der Befestigung (Auffädelung) gedient hat. Die Bruchlinie am Unterrand ist sehr glatt, ohne Kratzspuren. Möglicherweise ist es ein Anhänger mit natürlichem Bruch; es könnte aber auch sein, daß die Schale als Kratzer oder Schaber vorgesehen war und noch nicht verwendet worden ist. Die Länge des Stückes beträgt $51 \mathrm{~mm}$, es dürfte von einer adulten Schale stammen. - Abb. 14

Das zweite Fragment ist ein kleiner Teil des Vorderendes, Unterrand; möglicherweise von derselben Schale.

Der anthropologischen Bestimmung zufolge handelt es sich um einen Mann (19-30 Jahre); weitere Beigaben sind Keramikgefäße.

Verf. 262, Fnr. 7 (Akte 260/81: „Muschel aus Fnr. 6“ - Leichenbrand aus Urne)

Chondrula tridens: 1 adultes Exemplar

Unio pictorum: 6 Fragm. von zwei linken Klappen, inadult, inkrustiert

Unio cf. crassus: 1 Fragm. (Unterrand) einer linken Klappe, wahrscheinlich adult, inkrustiert

Unionidae: 1 nicht identifizierbares Fragment

Es handelt sich um ein männliches Individuum (anthropologische Bestimmung, 35-45 Jahre); weitere Beigaben: 1 Bronzeringlein, Keramikgefäße.

Verf. 263, Fnr. 7 (Akte 260/81: „Muschel unter Fnr. 3“ - Schale)

Unio crassus: 1 rechte Klappe, inadult, dünn, inkrustiert

Es handelt sich wahrscheinlich um eine weibliche Bestattung (anthropologische Bestimmung; 19-30 Jahre), dafür sprechen auch die beiden beigegebenen großen Bronzenadeln; weitere Beigaben: Keramikgefäße.

Verf. 568, Fnr. 6 (Akte 260/84: "gelochte Schnecken“ aus Fnr. 5 Leichenbrand aus Urne)

Zebrina detrita: 103 einfach gelochte Schalen (Derzeit ist keine nähere Beschreibung dieser Schalen möglich, da die Diagnose nur nach einer Skizze der Fundobjekte erfolgen konnte.)

Aufgrund der beigegebenen Schmuckteile wird eine weibliche Bestattung angenommen. Es handelt sich um Zierknöpfchen aus Bronze, kleine Knochenringe von einer Schmuckkette und Keramikgefäße.

Verf. 662, Fnr. 5 (Akte 260/84: „Muschel aus Fnr. 4“ - Schale)

Unio pictorum: 5 Fragm. einer linken Klappe

2 Fragm. einer rechten Klappe

Möglicherweise handelt es sich um ein Exemplar; den Fragmenten nach zu schließen, eher dünnschalig und großwüchsig; inkrustiert.

Der anthropologische Befund der Bestattung ergab ein 13- bis 18jähriges Individuum; eine Geschlechtsbestimmung war nicht möglich. Da neben 


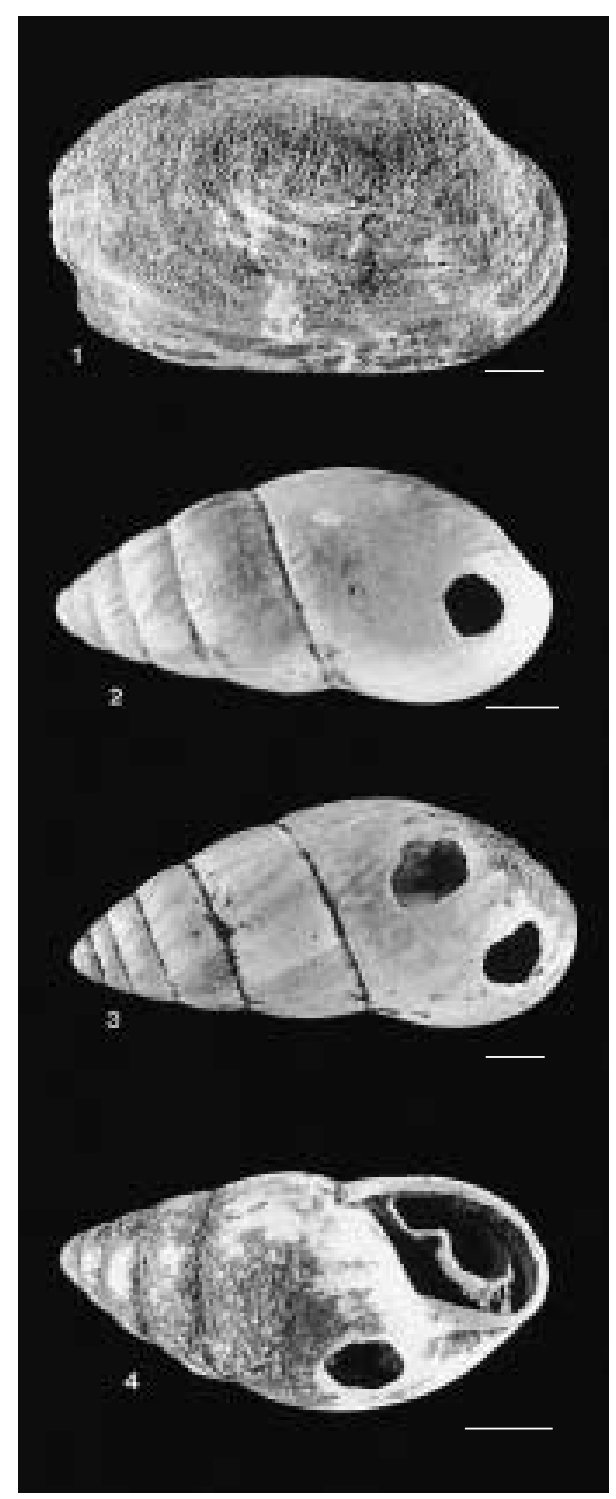

Abb. 1: Unio crassus PILIPSSON 1788, rechte Klappe (Verf. 723, Fnr. 3)

Abb. 2, 3, 4: Zebrina detrita

(O. F. MüLlER 1774) (Verf. 761, Fnr. 11)

Abb. 5, 6: Cepaea vindobonensis

(A. FÉRussac 1821) (Verf. 761, Fnr. 11)

\section{Maßstäbe:}

$5 \mathrm{~mm}$ (Abb. 1), alle übrigen: $3 \mathrm{~mm}$;

Fotos: H. GrILLITSCH (Univ. Wien)

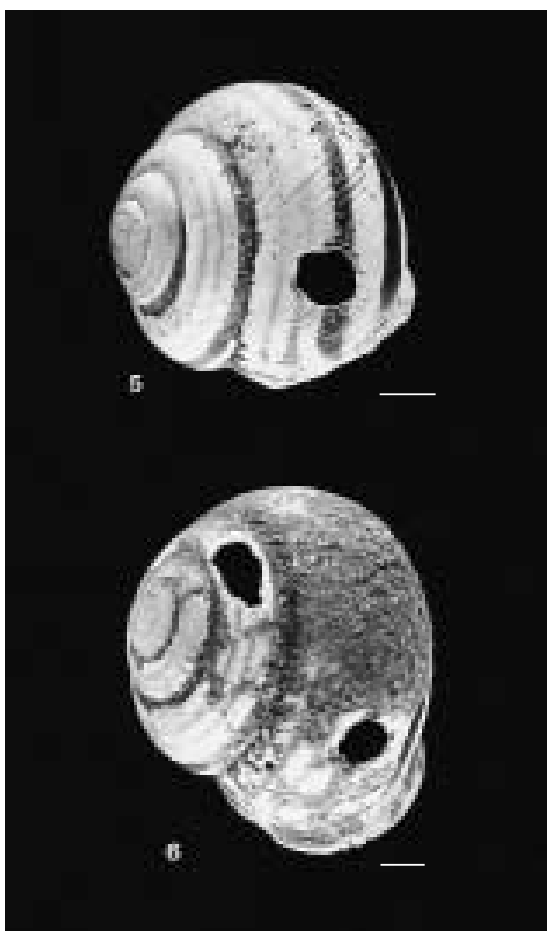

Keramikgefäßen auch ein Armreiffragment geborgen wurde, wird ziemlich sicher angenommen, daß es sich um eine weibliche Bestattung handelt.

Verf. 723, Fnr. 3 (Akte 260/84: „Muschel aus Fnr. 1“ - Urne)

Unio crassus: 1 rechte Klappe (am Hinterrand leicht fragmentiert, wahrscheinlich natürlicher Bruch; inkrustiert. - Abb. 1

Es handelt sich um ein Kind, 0-6 Jahre; keine Geschlechtsbestimmung möglich; weitere Beigaben: Keramikgefäße.

Verf. 761, Fnr. 11 (Akte 260/84: ,gelochte Schnecken“, „unter Fnr. 1“ - Urne)

Zebrina detrita: 155 fast durchwegs adulte Schalen: 148 Exemplare mit einer Lochung auf dem letzten Umgang (letztes Drittel knapp vor der Mündung; bei 11 Ex. ist der letzte Umgang an dieser Stelle abgebrochen), 7 Exemplare mit zwei Lochungen (bei $3 \mathrm{Ex}$. je einer Lochung knapp vor der Mün- 
dung und schräg oberhalb der Spindel, bei 4 Ex. zwei Lochungen auf der Rückseite des letzten Umganges). Abb. 2, 3, 4

Cepaea vindobonensis: 7 fast durchwegs adulte Schalen (5 Exemplare mit einer Lochung auf dem 3. Band knapp vor der Mündung, 2 Exemplare mit je einer Lochung auf dem letzten Drittel des letzten Umganges auf dem 3. Band bzw. auf dem vorletzten Umgang. Eines der einfach gelochten Exemplare an der Perforationsstelle ausgebrochen). - Abb. 5, 6

Die anthropologische Bestimmung der Leichenbrandreste ergab eine Frau (19-30 Jahre); weitere Beigaben: 1 Armreif, zerschmolzene Bronzefragmente, Keramikgefäße.

Um den Vergleich mit gegenwärtigen Schalen bzw. mit Meßdaten aus der Literatur zu ermöglichen, wurden 10 Zebrina detrita- und 5 Cepaea vindobonensis-Schalen vermessen.

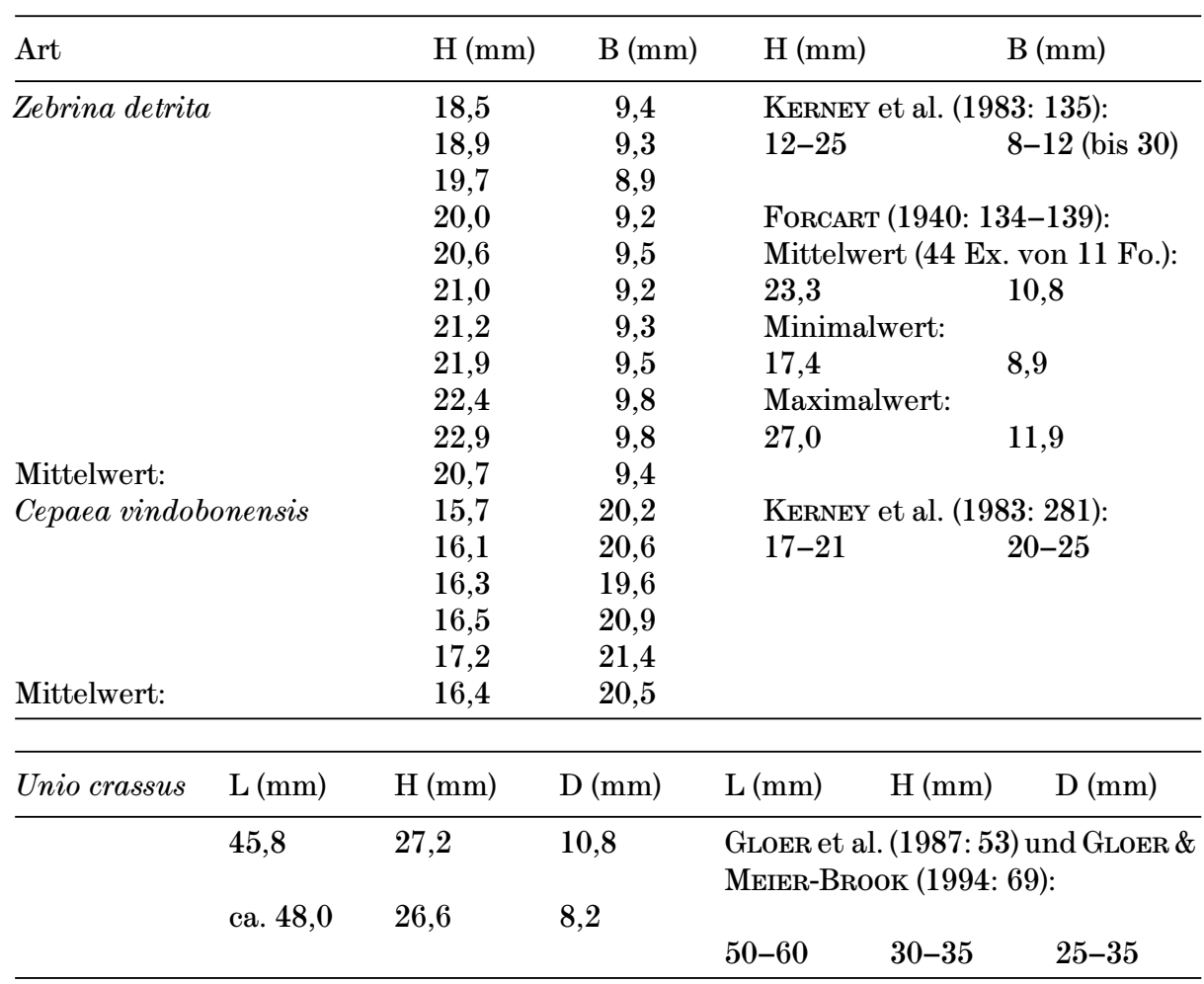

Die Zebrina detrita-Exemplare liegen somit im bekannten Normbereich, wenn auch der gerechnete Mittelwert unter dem von ForcarT erhaltenen liegt. Cepaea vindobonensis erscheint etwas flacher, auch Unio crassus ist etwas kleiner. Da bei dieser nur zwei meßbare Klappen vorliegen, sind diese Werte nur eingeschränkt zu betrachten.

\section{3. Über die Verwendung von Molluskenschalen als Schmuckobjekte}

Aus verschiedenen paläolithischen und neolithischen Fundstellen Österreichs liegen fossile und/oder etwa den Besiedelungszeiten entsprechende Molluskenschalen vor, die entweder unbearbeitet sind oder Bearbeitungsspuren aufweisen, die entweder auf eine Verwendung als einfache Geräte oder zu 
Schmuckzwecken hindeuten. Hier soll die letztere Verwendungsweise zur Diskussion gestellt werden:

Aus den ältesten Fundstellen des Gebietes (Gudenushöhle bei Nöhagen, Willendorf i. d. Wachau, Galgenberg bei Stratzing) liegt meist nur eine fossile Molluskenart vor, maximal zwei Arten (Schnecken, Dentalium sp.). Die Zahl der verschiedenen fossilen Arten steigt in den etwas jüngeren paläolithischen Fundstellen deutlich an (z. B. Grubgraben bei Kammern: 14 Arten, Kamegg: 12 Arten), wobei die Fossilien auch aus weiter entfernt liegenden Vorkommen, z. B. dem Wiener Becken, herbeigeschafft worden sind. Eine „Trendwende“ läßt sich im Neolithikum erkennen. Hier treten vor allem Spondylus-Klappen, die vermutlich aus dem Mittelmeerraum stammen und zu Schmuckstücken verarbeitet worden sind, auf. Sie dominieren noch bis ins mittlere Neolithikum (Armreifen, Halbreifen, zylindrische „Perlen“, Doppelknöpfe usw.); vgl. Simetsberger (1993), Steininger (1995), Thenius \& VÁvra (1996: 77-88).

Unter den Beigaben im linearbandkeramischen Gräberfeld von Kleinhadersdorf (VB Mistelbach, Flur „Marchleiten“) waren u. a. Spondylus-Anhänger (z. B. Grab Verf. 81), walzenförmige Spondylus-Perlen (Grab Verf. 22), Ketten aus Dentalium-Röllchen, zu Armreifen und Gürtelschließen verarbeitete Spondylus. Bei einer Kleinstkind-Bestattung (Grab Verf. 26) wurden dagegen in 2 Reihen kranzförmig um den Kopf liegende kleine „Schneckenschalen" gefunden, die als Besatz einer Kopfbedeckung oder von Zierbändern interpretiert wurden (Neugebauer-Maresch 1992, Neugebauer 1995: 42-46).

In der $12 \mathrm{~m}$ westlich der Doppelbestattung im Zentrum der mittelneolithischen Kreisgrabenanlage von Friebritz befindlichen Gruppe von Bestattungen fanden sich unter den reichen Beigaben ebenfalls Spondylus-Perlen (zeitliche Stellung: Anfang der Lengyel-Zeit; Neugebauer 1995: 58-63, NeUGEBAUER-MARESCH 1995).

Im Bereich der Fluren „Am Wald“ bzw. „Kirchfeld“ an der Grenze der Katastralgemeinden Asparn/Zaya und Schletz liegt eine großflächige Fundstelle der Linearbandkeramik, die seit 1983 untersucht wird. Außerdem liegen vereinzelte Siedlungsspuren der frühen Bronzezeit und der Urnenfelderzeit vor. Die Funde, die hauptsächlich der klassischen Notenkopfkeramik angehören, beinhalten auch 2 Spondylus-Perlen (WINDL 1997).

„Dentalium-Röllchen“ befanden sich im Fundgut aus der notenkopfkeramischen Siedlung von Franzhausen (BLESL 1995).

In Ratzersdorf (Industriegelände St. Pölten-Nordost) wurde eine ausgedehnte frühbronzezeitliche Siedlung entdeckt (Unterwölblinger Kultur(gruppe), Stufe Gemeinlebarn II), die wahrscheinlich bis in die mittlere Bronzezeit weiterbestand. Aus der notenkopfkeramischen Besiedlungszeit sind 12 Grabschächte erhalten, unter diesen einige mit Molluskenschmuck: Bei Bestattung Verf. 556 wurden u. a. eine Spondylus-Klappe mit V-Einschnitt und Theodoxus-Schalen gefunden, in Grabgrube Verf. 205 „Schmuckröhrchen aus Serpulidenschalen" und Spondylus-Perlen, in Verf. 2062 Spondylus-Klappen (Blesl \& Neugebauer 1999; Abb. 8: Die hier abgebildeten Schneckenschalen sind Theodoxus danubialis und stammen daher ziemlich sicher nicht „von Fossilfundstellen aus der Umgebung des Traisentales“ (zit: p. 53), sondern sie wurden aus der Donau oder der Traisen gesammelt). Die Spondylus-Klappen wurden vermutlich als Tauschobjekte bezogen, wahrscheinlich aus dem Mittelmeergebiet.

In einem hallstattzeitlichen Brandgräberfeld in Hohenau a. d. March (Hausbrunnerstraße) fand man unter den Beigaben vereinzelt "gelochte Schneckenhäuser" (NeUGEBAUER 1995: 106-108). 
Molluskenschalen-Schmuck aus jungpaläolithischen und frühmesolithischen Fundschichten Süddeutschlands wird von RäHLE (1978, 1981, 1983a,b, 1987, 1994) ausführlich beschrieben: Von mehreren Höhlen und Felslöchern liegen aus mesolithischen Kulturschichten Schmuckschnecken verschiedener Art und Herkunft vor (Jägerhaushöhle, Große Ofnet-Höhle, Nördlinger Ries, Bettelküche, Burghöhle Dietfurt, Propstfels bei Beuron, Falkensteinhöhle bei Thiergarten/Obere Donau, Zigeunerfels nahe Sigmaringen). Die Meeresmollusken sind vermutlich alle mediterranen Ursprunges; besonders beliebt dürfte Columbella rustica (LinnaEus) gewesen sein. Diese Art liegt auch aus anderen Fundstellen, u. a. aus dem obersten mesolithischen Horizont von Birsmatten-Basisgrotte (bei Basel) und aus meso- und neolithischen sowie frühbronzezeitlichen Schichten aus dem Abri de Châteauneuf-lez-Martigues (westl. Marseille) vor, sodaß der mögliche Transportweg von letzterem Fundgebiet rhônetalaufwärts bis in die Schweiz, ins obere Donautal und zum Nördlinger Ries geführt haben dürfte. Die Fossilien stammen vermutlich aus dem Mainzer Tertiärbecken und aus dem Steinheimer Becken bei Heidenheim. Auch die danubische Lithoglyphus naticoides kommt unter den Schmuckschnecken vor (Zigeunerfels, Große Ofnethöhle). Der Autor äußert die Vermutung, daß diese Art aus dem östlichen Mitteleuropa importiert worden sein dürfte, da sie im Altholozän in der Donau wahrscheinlich nur bis etwa Regensburg vorgedrungen ist.

Der überwiegende Teil des Schalenschmucks vom Hohlen Fels bei Schelklingen wird dem Magdalénien zugeordnet, aus dem Gravettienhorizont liegen nur 2 Fragmente vor. 1 Art (Littorina obtusata (LinNAEUs)), die auch gegenwärtig an den europäischen Atlantikküsten sehr verbreitet ist, verweist auf Handelsbeziehungen nach Westeuropa. Aus dem Westen dürften auch einige der Fossilien stammen (Mainzer, Belgisches oder Pariser Tertiärgebiet).

Viele Individuen kommen aus dem etwa $50 \mathrm{~km}$ von der Fundstelle entfernten Steinheimer Becken; die übrigen wahrscheinlich aus der Molasse des oberen Donaugebietes (vor allem Viviparus suevicus (WENz) scheint im Magdalénien Süddeutschlands als Schmuck sehr beliebt gewesen zu sein).

Die vielen durchbohrten Molluskenschalen aus dem Magdalénien vom Petersfels im Brudertal bei Engen/Hegau lassen ebenfalls weitreichende Beziehungen der dortigen Menschen erkennen. Es handelt sich überwiegend um festschalige Fossilien aus marinen und limnischen Tertiärablagerungen, dazu um einige wahrscheinlich aus dem Mittelmeer stammende Individuen. Die dem Petersfels nächstgelegenen Vorkommen der ersteren liegen im Mainzer Tertiärbecken, wobei fast alle Arten auch in weiter westlich gelegenen Tertiärgebieten vorkommen (belgisches und Pariser Tertiär). Die zweiteren (vor allem Viviparus suevicus) stammen wieder aus miozänen Süß- und Brackwasserschichten des oberen Donaugebietes. Bemerkenswerte Schmuckobjekte sind ferner einige Schwarzjura-Fossilien (Lias), deren dem Petersfels nächstgelegene Vorkommen etwa $25 \mathrm{~km}$ westwärts davon im Gebiet des Wutachtales sind. Zum sonstigen Fundinventar der Magdalénien-Stationen im Brudertal siehe auch ALBREcht (1995).

Die Schmuckschnecken aus den obersten mesolithischen Fundschichten des Helga Abri bei Schelklingen kommen offenbar aus dem Steinheimer Bekken (Gyraulus sulcatus (HILGENDORF)). Dieses ist auch teilweise das Herkunftsgebiet der Ensembles von Schalen aus jungpaläolithischen und frühmesolithischen Schichten des Abri Felsställe bei Mühlen (Alb-Donau-Kreis; vgl. KIND 1987). Die Mehrzahl der aus dem Mesolithikum letzterer Fundstelle vorliegenden Steinheimer Schnecken ist die flache, mehr scheibenförmige 
Gyraulus sulcatus; im Magdalénien ist Viviparus suevicus am häufigsten. Diese scheint überhaupt im Gebiet südlich der Schwäbischen Alb ein sehr charakteristisches Schmuckobjekt in Magdalénien-Stationen gewesen zu sein (etwa um Bölling und die Ältere Dryaszeit; s. oben). Ein (?) „Modetrend“ läßt sich auch bezüglich der Verwendung der Steinheimer Gyraulen erkennen: Während des Jungpaläolithikums und des Frühmesolithikums („Stufen Beuronien A und B“) wurden vor allem kegelförmige Schalen von Gyraulus trochiformis (STAHL) verwendet, im Frühmesolithikum („Stufe Beuronien C“) dagegen überwiegend die flachen, scheibenförmigen von Gyraulus sulcatus.

Über Molluskenschalen als Trachtbestandteile bzw. als Rohstoff für „Muschelperlen“ oder „Schmuckplättchen“ weiterer deutscher Fundstellen siehe u. a. Hertel (1956): Margaritifera auricularia (Spengler) und Margaritifera margaritifera (LINNAEUS) in sächsischen Ausgrabungen (Neolithikum und Bronzezeit), FaLKNER (1988): „Große Grube“ von Heidelberg-Neuenheim (rössenzeitlich; dominant Margaritifera auricularia, außerdem Unio crassus nanus LAMARCK, eine mesozoische Auster und die im westlichen Mittelmeer und im europäischen Atlantik vorkommende Glycymeris glycymeris (LiNNAEus), Spatz (1996): Nekropole von Trebur (Kr. Groß-Gerau, Hessen; mittelneolithisches Gräberfeld, belegt von der Hinkelstein- und Großgartacher Kultur); Spondylus-Perlen, Scheibenperlen aus Margaritifera auricularia; durchlochte fossile Schneckenschalen und Muscheln aus dem Tertiär des Mainzer Beckens bzw. rezente aus Mittelmeer oder Atlantik (Glycymeris); durchbohrte Klappen von Spondylus und Margaritifera auricularia als Gürtelschließen; DiECKMANN et al. (1997): jungsteinzeitliches Pfahlbaudorf von Hornstaad-Hörnle am Westufer des Bodensees (etwa 3900 v. Chr.); Schmuck aus Schalen mariner Mollusken, z. B. Columbella rustica, Dentalium-Perlen sowie einzelne fossile Schmuckschnecken aus dem Pariser Becken verweisen auch hier auf weitreichende Handelsbeziehungen.

Sehr interessant ist auch das von Terberger (1994) von einem Homo sapiens-Lagerplatz bei Wiesbaden beschriebene "Schmuckrohmaterial-Depot" (ca. 32.000 Jahre alt, Aurignacien-Kultur), mit „10 vollständigen Muscheln in intensiv mit rotem Ocker eingefärbtem Sediment". Die Individuen stammen aus nahegelegenen tertiären Sanden und könnten nach Ansicht des Autors ein wichtiger Grund für das Aufsuchen dieses Platzes gewesen sein.

Bemerkenswerte Informationen enthält die Darstellung der Linearkeramischen Gräberfelder Bayerns von NIEszery (1995) bezüglich der Bestandteile von Totentracht und Schmuck. Auf die „runde Spondylusklappe“ (Gürtelanhänger: Frauengräber), den „V-Spondylus“ (als Gürtel- oder Gewandschließen, typisch für einen älteren Abschnitt der jüngeren Bandkeramik), Spondylus- und Steinperlen, „Dentalien“- (= in Wahrheit Protula-Röhrenperlen) und Spondylus-Armringe (nur in Männergräbern) wird hier nicht näher eingegangen. Der Kopf- und Halsschmuck aus Süßwasserschnecken (vorwiegend Theodoxus danubialis (C. PFEIFFER), in einem Fall auch Theodoxus transversalis (C. PFEIFFER) und Lithoglyphus naticoides (C. PFEIFFER)) erscheint charakteristisch für die niederbayerische Bandkeramik. Besonders eindrucksvolle Fundlagen werden aus den Gräbern Ai 32, Ai 60 und Ai 143 (Gräberfeld Aiterhofen), Se 29 (Sengkofen) und Ma 6 (Mangolding) beschrieben: Ai 32 ist das Grab einer älteren Frau, bei der fast alle der über 200 TheodoxusSchalen unmittelbar auf dem Scheitel lagen (zit.: Abb. 100). Die ursprüngliche Anordnung der Schalen dürfte in mindestens 5 parallelen Reihen von der Stirn zum Hinterhaupt verlaufen sein; außerdem waren kleine Gehänge vor dem Oberkörper. Ähnlich, doch nicht so dicht, ist die Anordnung aus den Grä- 
bern Ai 143 und Se 29 (in mindestens 2 parallelen Reihen über den gesamten Schädel). Im Frauengrab Ai 60 sind die Schnecken zu geradlinigen Strängen geordnet, die in mindestens 3 konzentrischen Kreisen um den Hinterkopf laufen (zit.: Abb. 99). Die Schalen sind gegenüber der Mündung abgeschliffen und waren wahrscheinlich direkt auf eine Haube oder ein Haarband aus Stoff oder Leder genäht; im Grab Ai 60 bildeten sie vielleicht eine Art Haarnetz. Ein ähnlicher Befund wie der aus dem letzteren Grab liegt auch aus der Nekropole von Essenbach-Ammerbreite (Linearbandkeramik; Grab 27) vor. Aufgeschliffene Schneckenschalen, im Halsbereich liegend, kombiniert mit dem Kopfschmuck oder ohne ihn, lassen auf Halsketten oder Gehänge schließen, die zu einem Kopfschmuck gehörten und im Zuge des Verwesungsprozesses bis in die Halsgegend gefallen sind.

Die 4 durchbohrten Kaurischnecken, die aus einem Schacht der Dietersberghöhle bei Egloffstein (Kr. Forchheim) zusammen mit Glasperlen geborgen wurden, dürften als kultische Beigabe zu verstehen sein: Zwischen dem 7 . und 5. Jh. v. Chr. wurden mindestens 30 Personen mit Kleidung und Schmuck in diesen Schacht hinabgestürzt (KunTNER 1996).

Fossile tertiäre Schnecken (Melanopsis sp.) als Bestandteile von Halsketten und zahlreiche Schalen von Arianta arbustorum (LiNNAEus) wurden auch in der Dreifachbestattung der Mammutjäger von Dolní Věstonice (Mähren) gefunden (KLÍMA 1995). Die Landschneckenbeigabe dürfte hier aber ebenfalls kultischen Hintergrund haben, wenn auch die Abgrenzung von Schmuck- und Amulettbeigabe nicht immer möglich ist.

Über Molluskenschmuck aus jungpaläolithischen Fundstellen der Nordwestschweiz berichtet SEDLmEIER (1988; Hollenberghöhle 3, Höhle BirseckErmitage, Kohlerhöhle, Kastelhöhle, Rislisberghöhle, Höhle Käsloch, Abri Chesselgraben; mit Literaturübersicht). Die Molluskeninventare sind spätmagdalénien-zeitlich („Bereich Ältere Dryas/Beginn Alleröd“) und dokumentieren ebenfalls weitreichende Fernverbindungen zum Mainzer Tertiärbekken, nach Westeuropa, zum Mittelmeerraum bzw. in nordöstlicher Richtung in das obere Donaugebiet (Brackwassermolasse). Der kleine Abri von Vionnaz (Rhônetal, 15 km vom Genfer See entfernt) enthält eine Schichtfolge des älteren Mesolithikums (zwischen 9500 und 8000 BP). Die dortigen Jäger und Sammler handelten genauso Schmuck über weite Strecken hin: Die Schalen von Columbella rustica wurden offenbar von den Mittelmeerküsten rhôneaufwärts transportiert und als Anhänger gelocht und verwendet (СRotTI \& PIGNAT 1995; vgl. auch oben).

Als Beispiel für die Verwendung mariner Molluskenschalen als Schmuckobjekte in Osteuropa wird auf die Ausgrabung von Ayios Mamas (Chalkidike/ Nordgriechenland) verwiesen. Die Stratigraphie umfaßt hier eine Zeitspanne vom Ende des 4. vorchristlichen Jahrtausends bis ins 11.-12. Jh. n. Chr. Viele gut erhaltene Schalen verschiedener dort vorkommender Arten werden der mittleren Bronzezeit zugeordnet (zwischen 2000 und 1700 v. Chr.; BECKER 1996). Es handelt sich um Arten und Gattungen, die auch von anderen prähistorischen Fundstellen Griechenlands, vor allem auf Kreta, bekannt sind.

Verschiedene Paläolithstationen der Südwesttürkei, Umgebung von Antalya, wurden von Tübingen untersucht (ALBRECHT et al. 1992). Die „shell ornaments“ von Öküzini („oxen cave“, ${ }^{14} \mathrm{C}$ in $\mathrm{AH} 2$ : $8595 \pm 90 \mathrm{BP}$, in $\mathrm{AH} 17$ : $12190 \pm 270 \mathrm{BP}$ ) stammen aus den archäologischen Horizonten $\mathrm{AH} 13$ bis AH 2, wobei der Schmuck ähnlich wie der aus der nahegelegenen Höhle Karain B ${ }^{(14} \mathrm{C}$ : 12500 BP; RäHLE, unpubl.) aus marinen Arten der Mittelmeerküste, 
hauptsächlich Arcularia gibbosula LinnaEus, Columbella rustica und Dentalien besteht.

Das Weichtierschalen-Inventar israelischer Fundstellen wurde von MiENIS $(1989,1990,1992,1998)$ bearbeitet: Natufian site von Mallaha (Eynan; über 13.000 Individuen); $91 \%$ davon sind süßwasserbewohnende Arten aus der näheren Umgebung und vom Oberen Jordan. Die marinen Arten zeigen Handelsbeziehungen zum Mittelmeer und zum Roten Meer. Die Bearbeitungsspuren an vielen Individuen lassen annehmen, daß sie wahrscheinlich als Anhänger getragen worden sind. Relativ schlecht erhalten war das Fundgut aus Nahal Oren (Karmelgebirge; etwa 9500-6000 BP). Es sind hauptsächlich Landschnecken des Gebietes sowie wenige marine Arten. Die auffallende Besonderheit dieser Fundstelle sind die praktisch von Natur aus "fertigen“ Anhänger - die Opercula von Pomatias olivieri (CHARPENTIER), die nur noch in der Mitte gelocht wurden. - Die Kombination aus der byzantinischen Kirchenruine von Pisgat Ze'ev (Jerusalem), Glycymeris-Donax-Aspatharia, findet sich oft in Fundorten der Byzantinisch-früharabischen Periode. Auch hier dienten die gelochten Individuen wahrscheinlich als Anhänger. - Eine Brunnenfüllung aus Khirbet Ibreiktas (südlich der Hadera-West-Bahnstation) ist spätrömisch (3.-4. Jh.) und enthält wenige Individuen aus dem Mittelmeer (darunter ein Glycymeris-Anhänger) bzw. lokale terrestrische Arten.

Fundmaterial aus ägyptischen Ausgrabungen wurde von FALKNER (1981, 1982) bearbeitet: Aus dem Haupttempelkomplex von North Saqqâra (3 marine, 5 limnische Arten aus dem Nil; unter den marinen ist Monetaria annulus, mit künstlich abgeschliffenem Rücken - eine häufige Bearbeitungsform zum Aufnähen bzw. -flechten oder auf Schnüre knoten) sowie aus dem Satettempel von Elephantine (5.-11. Dynastie, etwa über die 2. Hälfte des 3. vorchristlichen Jahrtausends, 7 Arten, davon 4 marine aus der Mittelmeerfauna, die auch aus plio-pleistozänen Sedimenten aus Unterägypten stammen könnten. Darunter sind je ein Anhänger oder Kettenglied aus Hinia reticulata mamillata (RISso) und Glycymeris violascens (LAMARCK)).

\subsection{Interpretation des Fundgutes}

Man könnte annehmen, daß der Schmuck aus den dekorativen Zebrina- und Cepaea-Schalen einen lokalen „Modetrend“ der im Fundgebiet lebenden urnenfelderzeitlichen Frauen repräsentiert. Attraktiv an Zebrina ist sowohl die Schalenform, die entfernt zahnähnlich ist, als auch die unregelmäßige blaßrötliche bis violettbraune Längsstreifung bis -flammung, an Cepaea die helicoide Schale mit den dunkelbraunen Spiralbändern; in beiden Fällen ist die Grundfarbe weiß, schwach weißgrau oder - bei Cepaea - manchmal gelblich. Zebrina dürfte die vordergründig verwendete Art gewesen sein, sie muß also auch in ausreichenden Populationen vorgekommen sein. Während die einfach gelochten Individuen sehr wahrscheinlich aufgefädelt als Kette getragen worden sind, eventuell beide Arten kombiniert und alternierend (Abb. 7-10), auch Zebrina allein, gehörten die zweifach gelochten vermutlich dem Kleidungsbesatz an, d. h. sie wurden direkt aufgenäht (Abb. 11-13). Da nur wenige Individuen zweifach perforiert sind, könnte man weiter folgern, daß die Dekoration von Kleidungsstücken mit Schneckenschalen eher spärlich war oder daß die Schalen auch als eine Art (Zier-)Knöpfe gedient haben könnten.

Da der Erhaltungszustand der Schalen sehr gut ist, dürften sowohl der Schmuck, d. h. die Ketten, als auch die (?) Kleidung nachträglich dem Leichenbrand beigegeben worden sein. Ob diese Schnecken nur Bestandteile der 

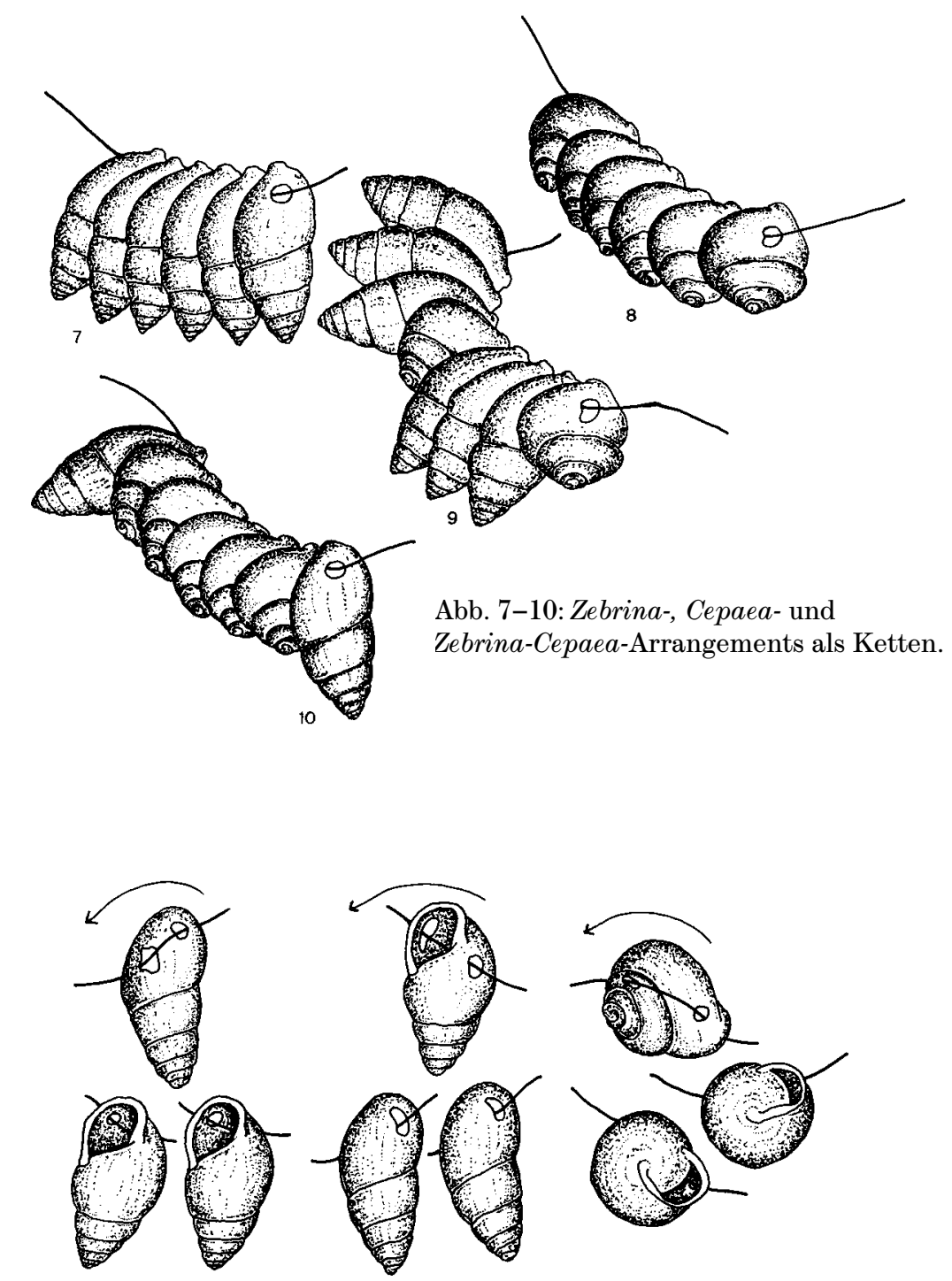

11

12

13

Abb. 11-13: Applikationsmöglichkeiten der zweifach gelochten Zebrina- und CepaeaSchalen an Kleidungsstücken.

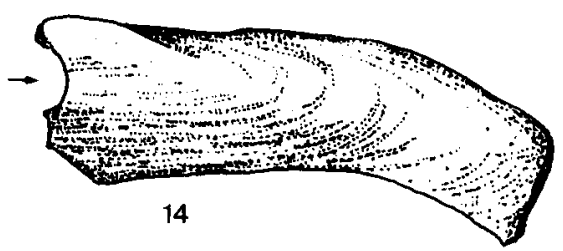

Abb. 14: Reste einer linken Klappe von Unio pictorum (Linnaeus 1758); Verf. 74, Fnr. 5. Unterhalb des Wirbels ist eine ausgebrochene Perforation sichtbar (Pfeil). Natürliche Größe.

weiblichen Tracht bzw. des Schmuckes waren, kann nicht gesagt werden. Auf geschlechtsspezifische Unterschiede im Spondylus-Schmuck weist NIESZERY (1995: 173-194, Abb. 95A, 95a, 95B) hin: Rund zugeschliffene SpondylusKlappen waren wahrscheinlich Gürtelanhänger bei Frauen, Spondylus-Armringe wurden dagegen von männlichen Individuen, fast immer auf dem linken Oberarm, getragen (Linearkeramische Gräberfelder in Bayern). 
Die Reste der Unio-Klappen bei den höchstwahrscheinlich weiblichen Bestattungen könnten symbolhaften Charakter haben (Muschelschale Weiblichkeitssymbol). Die Fragmente zeigten keinen Hinweis für eine eventuelle geräthafte Verwendung oder eine Lochung, die auf das Tragen nach Art eines Anhängers schließen ließe. Möglicherweise Symbol- oder Amulettcharakter hat auch die Einzelklappe von Unio crassus aus der Kinderbestattung (Verf. 723, Fnr. 3). Eine Beigabe als „Wegzehrung“ dürfte eher nicht anzunehmen sein, da in diesem Fall mehrere Individuen beigegeben worden wären. Das Fragment der linken Klappe von Unio pictorum (Verf. 74, Fnr. 5) könnte aufgrund der ausgebrochenen Perforation und des Unterrandes als ein Kratzoder Schabegerät, das an einer Schnur befestigt werden konnte, aber noch nicht gebraucht war, gedeutet werden (Abb. 14). Solche Geräte würden aber eher dem weiblichen Inventar zuzuordnen sein (Nahrungszubereitung, Auskratzen von Gefäßen, Glätten von Leder, Stoffen u. dgl.). Es wäre daher auch die Interpretation „Anhänger" möglich, mit natürlichem Bruch. Die UnioFragmente aus der Männerbestattung (Verf. 262, Fnr. 7) erlauben keine Interpretation. Auffallend ist jedenfalls, daß alle Klappenreste mit Ausnahme des genannten Unio pictorum-Fragments (Verf. 74, Fnr. 5) „naturbelassene“ Oberflächen zeigen, dieses jedoch völlig glatt, von Inkrustierungen befreit, erscheint. Diese Tatsache unterstützt auch die Deutung als Anhänger eher als die des Gebrauchsgegenstandes. Die Einzelschale von Chondrula tridens ist offenbar ein Lesefund und nicht als Beigabe zu verstehen.

Ein Blick auf die ökologischen Ansprüche der terrestrischen Arten läßt annehmen, daß warm-trockene, teils offene, teils gebüschbewachsene Standorte während der Besiedlungszeit in der Umgebung bestanden haben, vor allem Trocken- und Halbtrockenrasen. Für günstige Strömungsverhältnisse und sauberes, sauerstoffreiches Wasser von Traisen bzw. Donau spricht Unio crassus. Konform damit gehen auch die Theodoxus-Funde aus der notenkopfkeramischen Bestattung von Ratzersdorf (siehe Kapitel 2.3); die Art stellt vergleichbare Ansprüche an ihre Wohngewässer.

\section{ZUSAMMENFASSUNG}

Aus sieben Fundnummern des urnenfelderzeitlichen Gräberfeldes von Nußdorf ob der Traisen (NÖ) lagen Molluskenschalen vor, die bestimmt und ausgewertet wurden. Es sind insgesamt 272 Individuen, die drei Arten von Landschnecken und zwei Arten von Süßwassermuscheln angehören. Alle lebten im unmittelbaren Bereich des Fundgebietes, während dessen Besiedlungszeit. Die vordergründig gesammelte Art ist Zebrina detrita (MüLLER). Die Bearbeitungsspuren (Perforationen) an den Schneckenschalen werden analysiert und die wahrscheinlichen Verwendungsweisen - Schmuck und Kleidungsdekoration - diskutiert.

Die Unio-Klappen sind möglicherweise überwiegend amuletthafte Beigaben; das Fragment aus der Männerbestattung (Verf. 74, Fnr. 5) hatte möglicherweise Schmuckfunktion.

\section{SUMMARY}

About shell ornaments from Nußdorf ob der Traisen (Lower Austria)

All shells in question date back to the Uk-period and are of continental molluscs. Three species of terrestrial gastropoda and two of bivalvia were 
identified among the remains of the burial place. From the zoogeographical point of view, all of them occurred in the surroundings of the site, in the direct vicinity. With the exception of Unio crassus (PHILIPSSON), they are still living today in this region.

The total number of specimens is 272; Zebrina detrita (MüLLER) is predominating. The majority of the shells shows traces of manipulation - a small man-made perforation near the mouth, some of them a second one at the dorsal side of the last whorl or near the columella (Zebrina), or at the penultimate whorl (Cepaea). It seems that strings of (primarily) Zebrina and Cepaea were a local „fashion“ in this period and region. Perhaps, they have been very popular. Typologically and in comparison with other shell ornaments known from Austrian sites (mainly belonging to the palaeolithic and neolithic period), this find refers to none of them. According to my information, this is the first evidence that this combination has been used. Until yet, it is impossible to establish, if these ornaments were a component only of woman's wear.

Most of the Unio-valves might have served as something like conjuring means. The origins from the man's grave (Verf. 74, Fnr. 5) has possibly been used as shell pendant.

\section{DANKSAGUNG}

Ich bedanke mich sehr herzlich bei Dr. Michaela Lochner (Prähistorische Kommission, Österreichische Akademie der Wissenschaften, Wien), daß sie mir die Bearbeitung der Funde anvertraut hat; bei Herrn Mag. F. C. Stadler (Wien) für die Computerfassung des Manuskriptes und bei Frau Dipl.-Graph. H. Grillitsch (Biologiezentrum der Universität Wien) für die Anfertigung der Fotografien.

\section{LITERATUR}

Albrecht, G. (1995): Rentierjäger am Ende der Eiszeit. - Archäol. Dtschl., 1: 34-35; K. Theiß, Stuttgart.

Albrecht, G., Albrecht, B., Berke, H., Burger, D., Moser, J., RÄhle, W., Schoch, W., Storch, G., Uerpmann, H. P. \& Urban, B. (1992): Late Pleistocene and early Holocene finds from Öküzini: A contribution to the settlement history of the bay of Antalya, Turkey. - Paléorient, 18 (2): 123-141.

BECKER, C. (1996): Nourriture, cuillères, ornements ... Les témoignages d'une exploitation variée des mollusques marins à Ayios Mamas (Chalcidique, Grèce). - Anthropozoologica, 24: 3-17.

BLesL, CH. (1995): Die Siedlung der Notenkopfkeramik von Franzhausen im Unteren Traisental, Niederösterreich. - In: Aktuelle Fragen des Frühen und Mittleren Neolithikums in Mitteleuropa, Internat. ÖGUF-Symposium, 18.-22. 10. 1995, Kolpinghaus Poysdorf, NÖ; Exzerptbroschüre Österr. Ges. f. Ur- u. Frühgesch. und Stadtgemeinde Poysdorf, 3 pp., 2 Abb.

Blest, Ch. \& Neugebauer, J.-W. (1999): Bedeutende Ergebnisse bei den Rettungsgrabungen des Bundesdenkmalamtes im Raum St. Pölten im Jahre 1998. - Archäol. Österr., 10/1: 49-54; Wien.

Crotti, P. \& Pignat, G. (1995): Die letzten Jäger und Sammler der Schweiz. - Archäol. Dtschl., 4: 10-14; K. Theiß, Stuttgart.

Dieckmann, B., Maier, U. \& VoGt, R. (1997): Hornstaad-Hörnle, eine der ältesten jungsteinzeitlichen Ufersiedlungen am Bodensee. - Archäol. Dtschl., Sonderh. (1997): 15-21; K. Theiß, Stuttgart.

FALKNeR, G. (1981): Part 2: Mollusca. - In: The Sacred Animal Necropolis at North Saqqâra. - Egypt Exploration Soc., London, pp. 140-144. 
FaLKNer, G. (1982): IV. Molluskenfunde der Ausgrabungen des Deutschen Archäologischen Instituts Kairo im Satettempel auf Elephantine. - Münchner Ägyptologische Studien, 40: 152-172, Taf. 14-15; Berlin.

FaLKNeR, G. (1988): F. Die Muscheln aus der „Großen Grube“ von Heidelberg-Neuenheim. - Materialhefte z. Vor- u. Frühgesch. in Baden-Württ., 11: 109-116; 180; Abb. 1a; Stuttgart.

Forcart, L. (1940): Monographie der türkischen Enidae (Moll., Pulm.). - Verh. Naturforsch. Ges. Basel, LI(1): 106-263, 3 Taf.; Basel.

Frank, C. \& ReIschÜTZ, P. L. (1994): Rote Liste gefährdeter Weichtiere Österreichs (Mollusca: Gastropoda und Bivalvia). - In: Rote Listen gefährdeter Tiere Österreichs, Grüne Reihe des Bundesministeriums f. Umwelt, Jugend u. Familie, 5. Aufl.; 283-316; Graz.

Gloer, P. \& Meier-Brook, C. (1994): Süßwassermollusken. - 11. Aufl., 136 pp., Dtsch. Jugendbund f. Naturbeobachtung (DJN); Hamburg.

Glokr, P., Meier-Brook, C. \& Ostermann, O. (1987): Süßwassermollusken. - 6. Aufl., 86 p., Dtsch. Jugendbund f. Naturbeobachtung (DJN); Hamburg.

Hertel, R. (1956): Praehistorische Funde von Margaritifera auricularia Spglr. und Margaritana margaritifera L. in Sachsen. - Abh. Ber. Staatl. Mus. Tierkd. Dresden, 23: 1-7; Dresden.

HoRwitz, L. K. \& Mienis, H. K. (1998): Faunal remains from a roman well at Khirbet Ibreiktas. - Atiquot, XXXV: 60-64.

Kerney, M. P., Cameron, R. A. D. \& Jungbluth, J. H. (1983): Die Landschnecken Nordund Mitteleuropas. - 384 pp; Verl. Paul Parey, Hamburg u. Berlin.

KIND, C.-J. (Hrsg.) (1987): Das Felsställe. Eine jungpaläolithisch-frühmesolithische AbriStation bei Ehingen-Mühlen, Alb-Donau-Kreis. Die Grabungen 1975-1980. Forsch. Ber. Vor- u. Frühgesch. Baden-Württ., 23: 385 pp, 100 Taf., Kommiß. Verl./K. Theiß, Stuttgart. - Mit Beiträgen von Berke, H., Campen, J, Czarnetzki, A., Jaguttis-Emden, M., Owen, L., RÄhle, W., Rottländer, R. C. A., Schoch, W., Storch, G., Torke, W., Vilette, Ph.

KLemM, W. (1974): Die Verbreitung der rezenten Land-Gehäuse-Schnecken in Österreich. - Denkschr. Österr. Akad. Wiss, 117: 503 pp, Springer, Wien-New York.

KLIMÁ, B. (1995): Kunst und künstlerische Darstellung der mährischen Mammutjäger. In: Schamanenzauber und Eiszeitkunst; Katalogreihe des Krahuletz-Museums Nr. 14: 13-24; Eggenburg.

Kuntner, K. (1996): Die Welt ins Haus geholt: Vorgeschichtliches Glas. - Archäol. Dtschl., 1: 28-31; K. Theiß, Stuttgart.

LOCHNER, M. (1994): Späte Bronzezeit, Urnenfelderzeit. Aktueller Überblick über die Urnenfelderkultur in Ostösterreich. In: NeugebaueR J. W., Bronzezeit in Ostösterreich, Wissenschaftliche Schriftreihe Niederösterreich 98/99/100/101, 222p.

Mienis, H. K. (1986): The molluscs of the excavation of the Early Arabic Site of Sde Boquer: Some further remarks. - Levantina, 60: 657-662.

Mienis, H. K. (1990): The molluscs of the Natufian site of Mallahia (Eynan). - Le Natoufien ou les prémices du Néolithique au Levant, Valbonne, Juin 1989: 1 p. (Abstr.).

Mienis, H. K. (1992a): Molluscs from the excavation of a Byzantine church at Pisgat Ze'ev, Jerusalem, Israel. - Soosiana, 20: 21-24.

Mienis, H. K. (1992b): Shells from the excavation of a Byzantine-Early Arabic site in the Southern negev, Israel. - De Kreukel, 28 (1): 6.

Neugebauer, J. W. (1992): Die frühen Kelten im Osten Österreichs. - Archäol. Österr., 3/1: 17-28; Wien.

Neugebauer, J. W. (1995): Archäologie in Niederösterreich. Poysdorf und das Weinviertel. - Verl. niederösterr. Pressehaus, St. Pölten-Wien; 128 pp. (Mitarb. v. CH. Neugebauer-Maresch, Beitr. v. F. Parisch u. S. Verginis).

NeugebaueR, J. W. (1997): Großflächiger Landverbrauch - Vorbeugende Rettungsgrabungen. Ergebnisse für die prähistorische Archäologie am Beispiel des unteren Traisentales im Zentralraum Niederösterreichs. - Archäol. Österr., 8: 40-57; Wien.

Neugebauer-Maresch, Chr. (1992): Der bandkeramische Friedhof von Kleinhadersdorf bei Poysdorf, NÖ. - Archäol. Österr., 3/1: 5-11; Wien.

Neugebauer-Maresch, Chr. (1995): Friebritz, NÖ: Gräbergruppe im Zentrum der Kreisgrabenanlage vom Beginn der Bemaltkeramik. - In: Aktuelle Fragen des Frühen und Mittleren Neolithikums in Mitteleuropa, Internat. ÖGUF-Symposium, 18.- 
22. 10. 1995, Kolpinghaus Poysdorf, NÖ; Exzerptbroschüre Österr. Ges. f. Ur- u. Frühgesch und Stadtgemeinde Poysdorf, 1 pp.

NiEsZery, N. (1995): Linearbandkeramische Gräberfelder in Bayern. - Internat. Archäologie, 16: 404 pp, Verl. Marie L. Leidorf; Espelkamp (hrsg. v. Dobiat, C., \& LEIDorF, K.; mit Beiträgen von Breinl, L., CARli-Thiele, P. et al., Endlicher, G.).

RÄHLE, W. (1978): 23. Schmuckschnecken aus mesolithischen Kulturschichten Süddeutschlands und ihre Herkunft (Propstfels, Falkensteinhöhle, Burghöhle Dietfurt, Zigeunerfels, Große Ofnet). - In: Das Mesolithikum in Süddeutschland , 2: Naturwissenschaftliche Untersuchungen. - Tübinger Monographien zur Urgeschichte, 5(2): 163-168 (Hrsg.: Taute, W.).

RÄHLE, W. (1981): Schmuckschnecken aus jungpaläolithischen Fundschichten vom Hohlen Fels bei Schelklingen (Alb-Donau-Kreis) - Archäol. Korr.-Blatt., 11 (3): 179180, Taf. 42; Mainz.

RÄHLE, W. (1983a): Schmuck aus Schneckenschalen von der Magdalénien-Station Petersfels bei Engen (Hegau). - In: Naturwissenschaftliche Untersuchungen an Magdalénien-Inventaren vom Petersfels, Grabungen 1974-1976. - Verl. Archaeologica Venatoria, Inst. f. Urgeschichte d. Univ. Tübingen (hrsg. v. AlBRECHT, G., Berke, H., \& Poplin, F.): 154-160.

RÄHLE, W. (1983b): Die Mollusken der Grabung Helga-Abri bei Schelklingen mit einer Anmerkung zum Fund einiger mesolithischer Schmuckschnecken. - Archäol. Korr.-Blatt, 13 (1): 29-36; Mainz.

RÄHLE, W. (1987): Schmuck aus Molluskenschalen von dem Abri Felsställe bei Mühlen, Stadt Ehingen, Alb-Donau-Kreis. - In: KIND, C.-J., Das Felsställe. Eine jungpaläolithisch-frühmesolithische Abri-Station bei Ehingen-Mühlen, Alb-DonauKreis. Die Grabungen 1975-1980. - Forsch. Ber. Vor- u. Frühgesch. BadenWürtt., 23: 383-385; Stuttgart.

RÄHLE, W. (1994): Neue Funde jungpaläolitischen Schalenschmuckes vom Hohlen Fels bei Schelklingen, Alb-Donau-Kreis, und vom Petersfels bei Engen, Kreis Konstanz. - Fundber. Baden-Württ., 19 (1): 95-98; Stuttgart.

ReIschütz, P. L. \& SACKL, P. (1991): Zur historischen und aktuellen Verbreitung der Gemeinen Flußmuschel, Unio crassus PhILIPsson 1788 (Mollusca: Bivalvia: Unionidae), in Österreich. - Linzer biol. Beitr., 23 (1): 213-232; Linz.

SEDLMEIER, J. (1988): Jungpaläolithischer Molluskenschalen-Schmuck aus Nordwestschweizerischen Fundstellen als Nachweis für Fernverbindungen. - Archäol. Korr.-Blatt, 18: 1-6, Taf. 1; Mainz.

Simetsberger, B. (1993): Die fossilen Molluskenreste aus jungpaläolithischen und neolithischen Fundstellen in Niederösterreich und Burgenland, Österreich. - Diplomarbeit NAWI-Fak. Univ. Wien, 158 pp, 19 Karten, 50 Tafeln.

Spatz, H. (1996): Forschung Neolithikum: Hinkelstein und Großgartach - Kontinuität und Wandel. - Archäol. Dtschl., 3 (1996): 8-13; K. Theiß, Stuttgart.

Steininger, F. F. (1995): Fossile und rezente Molluskenreste aus Paläolith- und Neolithstationen Ostösterreichs. - In: Schamanenzauber und Eiszeitkunst; Katalogreihe des Krahuletz-Museums Nr. 14: 25-32; Eggenburg.

Terberger, T. (1994): Lagerplatz des frühen Homo sapiens sapiens bei Wiesbaden entdeckt. - Archäol. Dtschl., 3: 45-46; K. Theiß, Stuttgart.

Thenius, E. \& VÁvra, N. (1996): Fossilien im Volksglauben und im Alltag. - SenckenbergBuch 71; Verl. W. Kramer, Frankfurt/Main; 197 Abb., 179 pp.

WindL, H. (1997): Ein Fundplatz überregionaler Bedeutung aus dem Nordosten Niederösterreichs. - Archäol. Österr., 8: 34-39; Wien.

Vorgelegt von w. M. Herwig FriEsinger

in der Sitzung am 16. Juni 2000. 
